\title{
The benefits and costs of comparisons in a novel object categorization task: Interactions with development
}

\author{
Luc Augier • Jean-Pierre Thibaut
}

Published online: 20 April 2013

(C) Psychonomic Society, Inc. 2013

\begin{abstract}
We investigated how children use within- or between-category comparisons to generalize novel names for novel objects on the basis of a nonsalient dimension (texture) rather than a salient one (shape). Previous studies have not experimentally addressed the costs associated with comparisons. We conjectured that increasing the number of stimuli to be compared (and thus, converging evidence in favor of the target texture-based generalization), might not necessarily be beneficial, especially in young children (3- to 4-year-olds vs. 5- to 6-year-olds). Our results showed that more evidence in favor of texture (i.e., more within-category exemplars sharing the same texture) did not linearly increase texture-based choices in the same way for younger and older children. They also revealed that between-category comparisons gave rise to texture-based generalizations in both age groups. Overall, our results show that even though within- and between-category comparisons contribute to generalizations based on texture, they also generate cognitive constraints that interact with age.
\end{abstract}

Keywords Comparison load · Word extension ·

Categorization $\cdot$ Conceptual development

\section{Introduction}

One major challenge for children is to build a conceptual system that makes sense of the world. One difficulty is that

\author{
L. Augier $(\triangle) \cdot$ J.-P. Thibaut \\ LEAD - CNRS UMR 5022, Université de Bourgogne, \\ Pôle AAFE - Esplanade Erasme, \\ 21065 Dijon, France \\ e-mail: Luc.Augier@u-bourgogne.fr \\ J.-P. Thibaut \\ e-mail: jean-pierre.thibaut@u-bourgogne.fr
}

generalization of novel concepts frequently means going beyond obvious and easily accessible perceptual appearances in favor of nonsalient dimensions (conceptual or perceptual; see Rakison \& Oakes, 2003).

What might promote generalizations based on nonsalient dimensions? Several recent studies have suggested that the opportunity to compare items belonging to the same category provides an extremely powerful means for learning about the world (Namy \& Gentner, 2002). Comparisons contribute to the discovery of unifying nonsalient properties such as taxonomic commonalities (e.g., two objects belong to the category of furniture) or nonsalient perceptual properties (e.g., object textures) that would remain unnoticed if the objects were processed in isolation (e.g., Gentner \& Namy, 1999). This positive role of comparisons has been documented for a wide variety of stimuli and situations in both adults and children. In the case of children, this has been shown for object names (e.g., Gentner \& Namy, 1999; Graham, Namy, Gentner, \& Meagher, 2010; Namy \& Clepper, 2010; Namy \& Gentner, 2002), names for parts (Gentner, Loewenstein, \& Hung, 2007), action verbs (Childers, 2011), adjectives (Waxman \& Klibanoff, 2000), and perceptual categories (e.g., Hammer, Diesendruck, Weinshall, \& Hochstein, 2009; Thibaut, 1991).

Typically, experiments involve comparison and nocomparison conditions, most often in the case of familiar stimuli. For example, in a novel name generalization task, Gentner and Namy (1999) pitted a taxonomic match against a perceptual match. In the comparison condition, children extended the novel name that was given to the exemplars (i.e., the standards; e.g., a bicycle and a tricycle) to a perceptually different taxonomic match (e.g., a skateboard), whereas in the no-comparison condition (e.g., a bicycle), they extended the name of the standards to the same-shape match (e.g., eyeglasses). Recently, Graham et al. (2010) replicated this finding for categories of unfamiliar stimuli. The target unifying dimension was the nonsalient dimension texture. In 
the comparison condition, preschoolers generalized the novel name to the stimulus with the same texture as the two standards, rather than to the stimulus that shared the same shape as one of the two standards. In the no-comparison condition, most 4-year-olds generalized the novel name to the shape match.

In the present study, we used the same novel name generalization task as in the studies referred to in the previous paragraph. However, our study specifically raised the issue of the cognitive costs of comparisons. Our key hypothesis was that increasing the number of stimuli involved in the task would increase the number of comparisons, which, in turn, would increase the cognitive costs of the task. Since young children have less developed executive functions (e.g., Anderson, 2002; see below), they might fail to integrate all the information available.

\section{Within- and between-category comparisons}

We also studied the respective contribution of within- and between-category comparisons (hereafter, contrast-category comparisons, which are comparisons involving stimuli from different categories) on categorizations of multidimensional stimuli. Both kinds of comparison increase the evidence in favor of the relevant dimension. According to Hammer, Bar-Hillel, Hertz, Weinshall, and Hochstein (2008), within-category commonalities (e.g., all the daxes have property $i$ and differ on their other properties) are clearly relevant in categorization in the sense that they contribute to the ability to distinguish categories. Hence, comparing stimuli belonging to the same category (e.g., several daxes) might highlight common properties.

However, comparisons may also involve stimuli from different categories. In the latter case, properties that are common to stimuli from different categories (e.g., "daxes and blickets have property X") do not differentiate between these categories. Indeed, being told that " $X$ is a $\operatorname{dax}$ and $Y$ is not a dax," children can infer that featural commonalities between $X$ and $Y$ are not sufficient to define what a dax is. Hammer et al. (2008) claimed that common properties of contrast categories are not very informative because knowing that one attribute does not discriminate between two categories does not tell which attribute might discriminate them except when the number of attributes defining the categories is very small. However, they might push them to further explore the stimuli in order to find properties that differentiate categories (see Hammer et al., 2008; see also Clark, 1993, for a discussion of the role of contrast in language learning). For example, in the context of novel adjective learning, Waxman and Klibanoff (2000) showed that 3 -year-olds were able to associate an object property (e.g., transparency) with an adjective (e.g., blickish) when the object displaying that property (e.g., a transparent plate) was contrasted to an object from the same category without that property (i.e., an opaque plate).

Recently, Namy and Clepper (2010) studied the role of contrast items in the aforementioned novel name paradigm (Gentner \& Namy, 1999). The comparison-plus-contrast condition featured two standards (i.e., two blickets) and a contrast object (a nonblicket). The contrast object and the two standards had the same - hence, not distinctive — shape (e.g., the two standards were a bicycle and a tricycle; the contrast object was barbells). The authors replicated the main effect of comparison and found a limited effect of contrast only when there were two standards. Most likely, knowing that shape was irrelevant did not tell participants which among the remaining dimension(s) was or were actually relevant (see Hammer et al., 2008).

\section{The present study}

Previous studies have demonstrated the positive effects of comparison on novel name generalizations. However, they have neglected the cost of comparisons. Our central hypothesis is that these comparisons generate cognitive costs that will be handled more or less efficiently by executive functions, such as working memory, inhibition, and cognitive flexibility (e.g., Zelazo, Carter, Reznick, \& Frye, 1997). In our comparison situations, increasing the number of standards belonging to the same category increases the number of comparisons that must be performed and held in working memory. Salient common perceptual attributes (such as shape) must be inhibited in order to find less salient unifying dimensions (such as texture). Shifting to a new dimension when a salient one, such as shape, is irrelevant for categorization requires cognitive flexibility. In this context, authors have defined the cognitive complexity of tasks. For example, Andrews and Halford (2002) defined it in terms of the number of sources of variation that are related and must be processed in parallel (see also Zelazo \& Frye, 1998, for a similar cognitive metrics). Given that executive functions are known to develop (e.g., Anderson, 2002), we hypothesized that comparison costs might differentially influence different age groups, which motivated the inclusion of two age groups.

We used unfamiliar categories defined by the nonsalient dimension texture as in Graham et al. (2010). Stimuli were constructed around two dimensions, texture and shape. In order to vary the processing load, we manipulated the number of standards (one, two, and four) and the presence of an item belonging to another category (no or one contrast item) that had the same shape as one standard stimulus. Adding more items, either standards or contrast items, increases 
texture relevance, decreases shape relevance, but increases cognitive costs. Thibaut, French, and Vezneva (2010) made a similar point in the case of analogies when they increased the number of related distractors, which were supposed to increase the inhibition and flexibility costs.

We compared contrast and no-contrast conditions. As was previously mentioned, a contrast object (e.g., "this is not a dax") tells that the dimension value it shares with the dax category (e.g., a given shape) is not diagnostic. However, repetition of this nondiagnostic dimension value (see Fig. 1) also potentially increases its saliency, even though the comparison should lead participants to discard it. Therefore, children have to inhibit it, a process that might be harder in the younger age group. Recently, Hammer et al. (2009), working with multidimensional artificial categories, found that 6-year-olds failed to integrate contrast information. This was not the case for adults (see also Andrews, Livingston, \& Kurtz, 2011, for adults). On these grounds, we predicted that younger children might not benefit from contrast conditions as older children would. In terms of inhibition and flexibility, the within- and the contrastcategory comparisons are interesting because they have opposing interpretations. Within-category commonalities lead to choice of the common dimension (here, texture), whereas contrast-category similarities lead to the rejection of the common dimension (here, shape).

We also compared two subclasses of within- and contrast-category comparisons that were defined around two stimuli: the 1-1 (i.e., one standard and one contrast item) and 2-0 (i.e., two standards and no contrast item) conditions. The question was whether, in the $1-1$ condition, the contrast item would lead to an increase in the use of texture (against the irrelevant shape) in the same way as in the 2-0 condition. In the latter case, differences in shape within the category should highlight texture as the unifying feature.

Finally, in our context of executive functions, age is a crucial factor. Although previous studies have shown that 4-year-olds can benefit from comparison, the question of how 4- and 6-year-olds will differentially integrate more comparisons remains open.

\section{Method}

Participants

A total of 216 preschoolers were tested individually at school. Two age groups were recruited. The younger children $(n=108$; mean age $=48.8$ months, $S D=6$, range: $38-58$ months $)$ and the older children $(n=108$; mean age $=66.3$ months, $\mathrm{SD}=5.5$, range: 59-77 months) were randomly assigned to one of the six experimental conditions, with 18 children per condition. Informed consent was obtained from their school and their parents.

Design

Younger and older groups of children were presented with novel object sets in one of six between-subjects experimental conditions resulting from the crossing of the variables number of standards (one, two, or four standards) and contrast (zero vs. one contrast) (see Fig. 1).

\section{Materials}

Seven sets of seven artificial grayscale objects pictured on cards were created. The dimensions of the cards were $12 \times 9$ $\mathrm{cm}$ (width $\times$ height), and the dimensions of the objects pictured on the cards were approximately $6 \times 6 \mathrm{~cm}$. Each participant completed two practice trials and five experimental trials. The objects' textures and shapes were chosen to be distinctive. Textures and shapes used on one trial differed from those used on other trials. There were two standards in the 2-0 and 2-1 conditions and four in the 4- 0 and 4-1 conditions. The standards shared the same texture but had different shapes. The contrast object (in the 2-1 and 4-1 conditions) had the same shape as one of the standards. The first test object, the shape match, had the same shape as one of the two standards (and as the contrast object) but differed in terms of its texture. The second test object, the texture match, had the same texture as the standards (and a different texture than the contrast object). In the 1-0 and 1-1 conditions, the standard had the same shape as the shape match (and the contrast object) and the same texture as the texture match. Each set was associated with one of 7 two-syllable novel names: youma, buxi, dajo, zatu, sepon, xanto, or vira.

\section{Procedure}

The experiment started with two practice trials, which were followed by five test trials presented in a random order. Each standard was introduced with a novel count noun (e.g., "this is a buxi" and "this is a buxi TOO" for the other standards). In the contrast conditions, a contrast object was introduced below the standard(s) as a nonmember of the category (e.g., "this is NOT a buxi"). The objects were presented sequentially and left in view. In the comparison conditions, stimuli were presented in a row, and their location was determined randomly. The forced choice test phase was identical in all conditions. The two test objects (i.e., the shape and the texture match) were introduced, and the child was asked to point to the one that was also a member of the category (e.g., "Show me which one of these two is also a buxi"). 
Fig. 1 Sample stimulus set and instructions used in the six experimental conditions that crossed the two factors: number of standards (one, two, or four standards) and contrast (zero or one contrast)
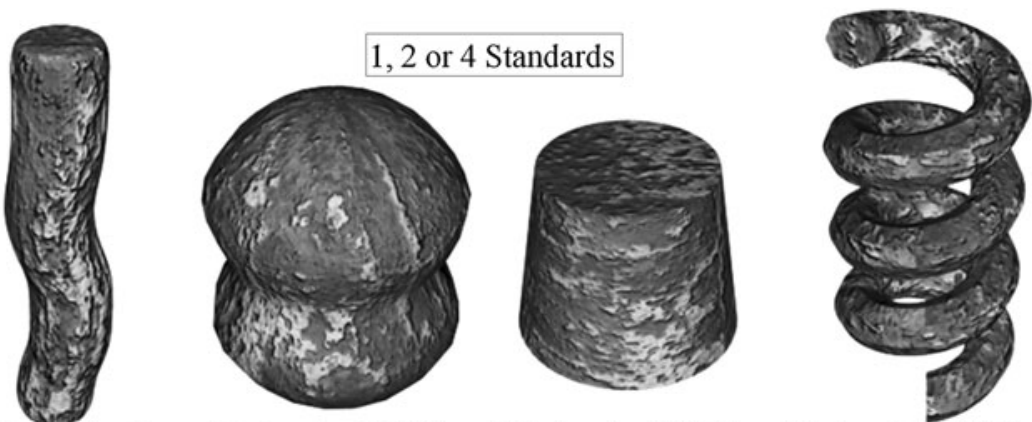

« this is a buxi» « this is a buxi TOO» « this is a buxi TOO» « this is a buxi TOO»

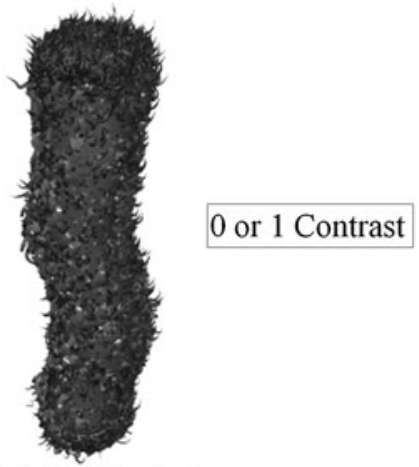

« this is NOT a buxi » texture match

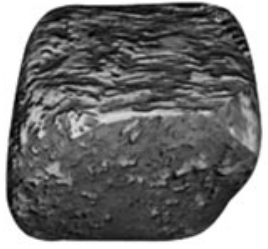

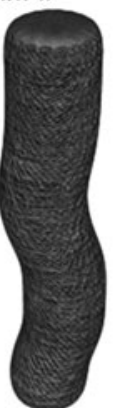

shape match
«Show me which one of these two is ALSO a buxi»

\section{Results}

Analyses were performed on the mean percentage of texture choices and on consistency patterns (see below). A 3 (number of standards) $\times 2$ (contrast) $\times 2$ (age) ANOVA was conducted on the percentage of texture choices. Older children chose the texture match significantly more frequently $(58 \%)$ than did younger children $(49 \%), F(1,204)=4.82, p<.05, \eta_{P}^{2}=.023$. The main effect of number of standards was significant, $\left.F(2,204)=54.97, p<.001, \eta_{P}^{2}=.35\right)$, with $26 \%, 58 \%$, and $77 \%$ of texture choices in the one-, two-, and four-standard conditions, respectively, showing that more standards meant more texture choices. These two main effects were subsumed by a marginally significant age $\times$ number of standards interaction, $F(2,217)=2.94, p=.055, \eta_{P}^{2}=.02$. The REGWQ post hoc procedure (see Howell, 2007) revealed that, in both age groups, the one-standard condition significantly differed from the two- and four-standard conditions. It also revealed that the four-standard condition was significantly higher than

the two other conditions in the older group only. Furthermore, older children performed significantly better than younger children in the four-standard case $(\mathrm{p}<.05)$ (see Fig. 2).

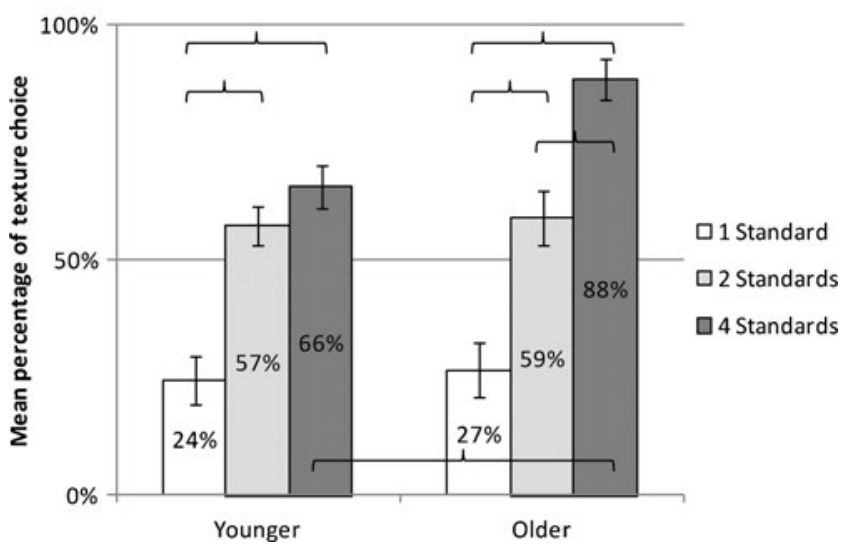

Fig. 2 Mean percentage of texture-based responses $(+S E M)$ as a function of age and number of standards. Braces show significant differences between columns $(p<.05)$ 
Overall, this interaction shows that increasing the number of standards benefited younger children less than it did older children.

The number of standards $\times$ contrast interaction was significant, $F(2,217)=3.55, p<.05, \eta_{P}^{2}=.03$ (see Fig. 3). The REGWQ post hoc procedure $(p<.05)$ revealed the following hierarchy: $1-0(17 \%)<1-1(34 \%)<2-1(54 \%)=$ $2-0$ (62\%), 2-1<4-0 (73\%), 2-0 = 4-0, 2-0<4-1 (81\%), $4-0=4-1$. In sum, there are three important results. First, the comparison between 1-0 and 1-1 showed that children benefited from a contrast-category comparison; second, there was a difference between $1-1$ and $2-0$, suggesting that withincategory comparisons are more powerful than contrastcategory comparisons when the comparisons involve two stimuli; third, there was a significant difference between the $2-1$ and the $4-1$ conditions, and no difference between 2-0 and 4-0 (see the Discussion section for an interpretation of this interaction).

Finally, we performed chi-square tests of independence corrected for multiple comparisons (Bonferroni) $(p<.05)$ on patterns of consistency (see Fig. 4). Children were categorized as texture consistent when they chose at least four texture matches (out of five), shape consistent when the shape match was chosen at least four times, or nonconsistent in the other cases. Indeed, a given percentage (e.g., 50\%) of shape choices can result from a majority of consistent participants (i.e., $50 \%$ shape-consistent and 50\% texture-consistent participants) or from a majority of inconsistent participants.

Importantly, there were fewer texture-consistent children overall in the $1-0(0 \%$ for both groups) than in the $1-1$ condition ( $22 \%$ for younger and $28 \%$ for older children). This shows that contrast-category comparisons alone contribute to highlighting texture and/or decreasing the dominance of shape.

As is shown in the analysis discussed above, only the older group benefited from more comparison items. There

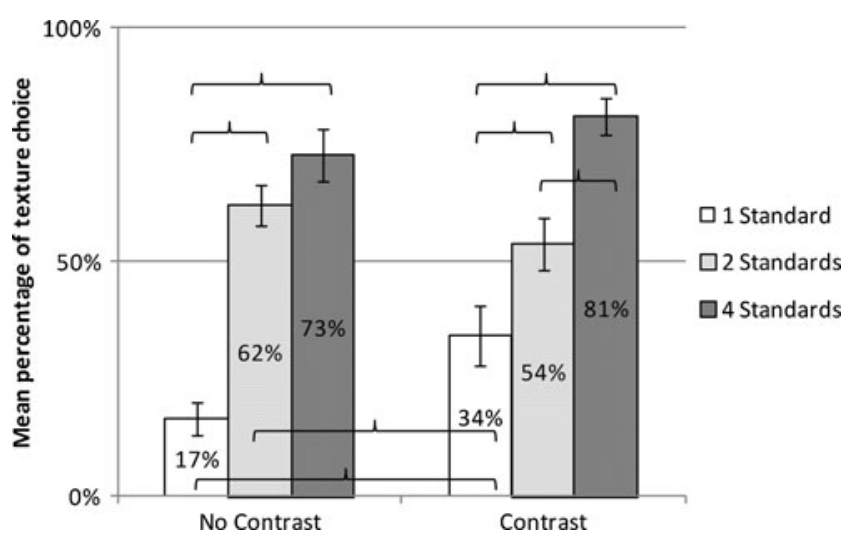

Fig. 3 Mean percentage of texture-based responses $(+S E M)$ as a function of contrast and number of standards. Braces show significant differences between columns $(p<.05)$ were significantly fewer texture-consistent older children in the $2-0(44 \%)$ and $2-1(50 \%)$ conditions than in the 4-0 (83\%) and 4-1 (94\%) conditions, respectively. Comparisons between the two age groups showed that there were significantly fewer younger than older textureconsistent children in the $2-1(50 \%$ and $11 \%$, respectively), 4-0 (83\% and 39\%, respectively), and $4-1$ ( $94 \%$ and $44 \%$, respectively) conditions.

\section{Discussion}

Previous studies have demonstrated the positive effects of comparison on novel name generalization. In the executive function framework, we hypothesized that the number of stimuli to be compared and the nature of the comparisons (within- or contrast-category) might matter. Indeed, presenting more stimuli to be integrated increases the number of comparisons and, thus, generates cognitive costs. More specifically, it was hypothesized that children of different age groups would not integrate the information in the same way because of differences in terms of executive function maturation (Zelazo et al., 1997). The results confirmed our predictions.

The discovery of a nonsalient dimension: Role of the number of standards

The interaction between age and number of standards, as well as the consistency patterns, showed that the younger group, overall, obtained similar results in the two- and four-standard conditions, whereas, for the older group, increasing the number of standards increased the percentage of texture-based generalizations (see Figs. 2 and 4). This shows that younger children make sense of the comparisons but do not integrate the extra information in favor of texture; they might compare two or three stimuli, but not all of them.

Within- and contrast-category comparisons

Regarding the two types of comparisons, two questions were raised. First, do contrast-category comparisons contribute to texture-based generalizations, and, second, is the information coming from contrast exemplars more difficult to integrate than within-category comparisons?

The difference between the 1-0 and 1-1 conditions addresses the first question. It shows that the contrast condition (1-1) resulted in more texture-based choices and more texture consistency than did the absence of contrast (1-0). To the best of our knowledge, this is the first evidence with young children that contrast-category comparisons alone can promote generalization based on a nonsalient dimension. Indeed, with familiar categories, Namy and Clepper (2010) 
Fig. 4 Percentage of children, for each experimental condition and for each age group, who were either texture consistent, shape consistent, or nonconsistent

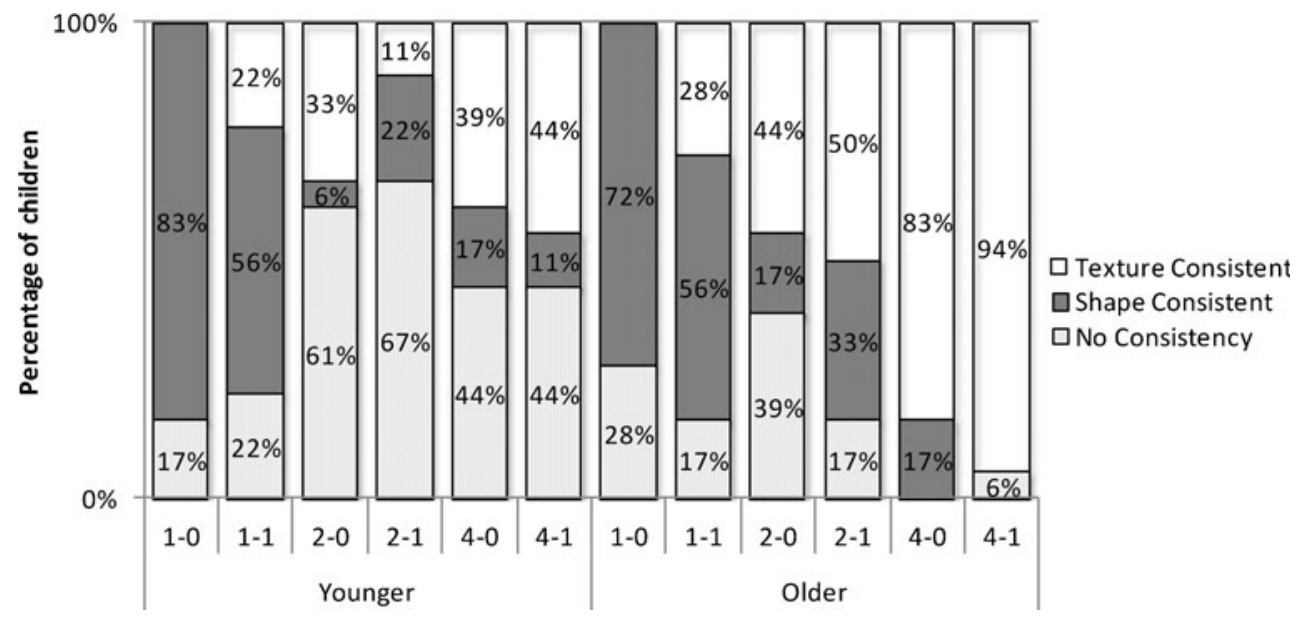

did not find any significant effect of contrast in their onestandard condition.

The answer to the second question"How did within- and contrast-category comparisons influence generalizations?"is that contrast-category comparisons gave rise to fewer texture-based generalizations. First, and importantly, the interaction between contrast and number of standards shows that the 1-1 condition generated fewer texture choices than did the 2-0 condition. Second, the consistency analysis shows that the $2-1$ condition generated significantly more difficulties in the younger group than in the older group, whereas this difference between the two groups did not exist in the 2-0 condition. As is shown in Fig. 4, this is due to a decrease in the percentage of texture consistencies in the younger group. This suggests that the younger group found it harder to integrate the extra information provided by the contrast item (2-1).

Overall, the present results show that contrast-category comparisons provide relevant information for texture-based generalization (1-0 vs. $1-1)$, even though it is more difficult to integrate than within-category comparisons (1-1 vs 2-0 and the difference between the age groups for 2-1). As was mentioned in the Introduction (see Hammer et al., 2008), within-category comparisons provide cumulative evidence in favor of the common dimension value (here, same texture) and indicate that nonrepeated dimension values (here, same shape) are irrelevant. By contrast, the contrast-category comparisons demonstrate that contrastcategory similarities (i.e., common shape) are irrelevant and, thus, that another dimension should be looked for but does not indicate which one. Another difficulty with contrast-category similarities is that the repetition of a common dimension value (same shape) increases its saliency but indicates that it is irrelevant and, thus, must be inhibited, as in the $1-1$ condition. The $2-1$ condition is more difficult, in this respect, because there are two types of commonalities (contrast-category for shape and within-category for texture) that children must interpret in opposite ways (i.e., reject shape vs. favor texture). In our case, this requires both inhibition capacities ("inhibit the salient common shape") and cognitive flexibility ("search for another dimension unifying the category"). The younger group's difficulties in $2-1$, together with the fact that they benefit less from more standards, are consistent with the general observation that cognitive monitoring (and thus, executive functions) is less developed in younger children, (e.g., Zelazo et al., 1997). This explanation also fits with Hammer et al.'s (2009) results showing that even 6- to 9-year-olds failed to integrate contrast items in a category learning task.

\section{The dynamic of comparisons}

Gentner and colleagues have argued that comparisons lead to generalization by structural alignment, which increases the saliency of less obvious within-category dimensions (e.g., Gentner \& Namy, 1999). Incorporating contrastcategory comparisons in alignment processes, as we did in the present experiment, shows that comparisons not only highlight alignable common within-category features and/or downplay alignable within-category differences (shape), but also downplay alignable common contrast-category features (shape) and/or highlight alignable distinctive contrastcategory features (texture).

In this context, increasing the number of standards decreases the relevance of alignments based on the a priori salient but irrelevant dimension (shape). Since decreasing shape saliency does not guarantee that unifying but less salient dimensions will be found, it becomes necessary to search for other dimensions on which the stimuli might be aligned. This is easier when more stimuli with the same dimension value are provided. However, as we said before, providing more items means coordinating more comparisons within the set and also between each standard and both matches, which was more difficult for the younger group.

To conclude, our main question focused on whether children would be able to integrate information from a growing 
set of comparisons involving within- and contrast-category information. In general, our data suggest that, even though both groups benefited from comparisons, younger children were less able to integrate all the available information. This suggests that comparisons generate their own cognitive costs, which must be taken into account when a comparison situation is devised.

Acknowledgments The authors wish to thank the Conseil Régional de Bourgogne for its financial support (Ph.D. grant to the first author), Faber Program. We thank the children who participated in the study, their parents, and the school staff for their assistance and Laurent Bergerot for his help in producing the stimuli.

\section{References}

Anderson, P. (2002). Assessment and development of executive function (EF) during childhood. Child Neuropsychology, 8(2), 71-82.

Andrews, G., \& Halford, G. S. (2002). A cognitive complexity metric applied to cognitive development. Cognitive Psychology, 45, 153-219.

Andrews, J. K., Livingston, K. R., \& Kurtz, K. J. (2011). Category learning in the context of co-presented items. Cognitive Processing, 12(2), 161-175.

Childers, J. B. (2011). Attention to multiple events helps two-and-ahalf-year-olds extend new verbs. First Language, 31(1), 3-22.

Clark, E. V. (1993). The lexicon in acquisition. New York, NY, US: Cambridge University Press.

Gentner, D., Loewenstein, J., \& Hung, B. (2007). Comparison facilitates children's learning of names for parts. Journal of Cognition and Development, 8(3), 285-307.
Gentner, D., \& Namy, L. L. (1999). Comparison in the development of categories. Cognitive Development, 14(4), 487-513.

Graham, S. A., Namy, L. L., Gentner, D., \& Meagher, K. (2010). The role of comparison in preschoolers' novel object categorization. Journal of Experimental Child Psychology, 107(3), 280-290.

Hammer, R., Bar-Hillel, A., Hertz, T., Weinshall, D., \& Hochstein, S. (2008). Comparison processes in category learning: From theory to behavior. Brain Research, 1225, 102-118.

Hammer, R., Diesendruck, G., Weinshall, D., \& Hochstein, S. (2009). The development of category learning strategies: What makes the difference? Cognition, 112(1), 105-119.

Howell, D. C. (2007). Statistical methods for psychology (6th ed.). Belmont, CA: Wadsworth Publishing.

Namy, L. L., \& Clepper, L. E. (2010). The differing roles of comparison and contrast in children's categorization. Journal of Experimental Child Psychology, 107(3), 291-305.

Namy, L. L., \& Gentner, D. (2002). Making a silk purse out of two sow's ears: Young children's use of comparison in category learning. Journal of Experimental Psychology. General, 131(1), 5-15.

Rakison, D. H., \& Oakes, L. M. (2003). Early category and concept development : making sense of the blooming, buzzing confusion. Oxford: Oxford University Press.

Thibaut, J.P. (1991). Récurrence et variations des attributs dans la formation des concepts. Unpublished doctoral thesis, University of Liège, Liège.

Thibaut, J. P., French, R., \& Vezneva, M. (2010). The development of analogy making in children: Cognitive load and executive functions. Journal of Experimental Child Psychology, 106(1), 1-19.

Waxman, S. R., \& Klibanoff, R. S. (2000). The role of comparison in the extension of novel adjectives. Developmental Psychology, 36(5), 571-581.

Zelazo, P. D., Carter, A., Reznick, J. S., \& Frye, D. (1997). Early development of executive function: A problem-solving framework. Review of General Psychology, 1(2), 198-226.

Zelazo, P. D., \& Frye, D. (1998). Cognitive complexity and control: II. The development of executive function in childhood. Current Directions in Psychological Science, 7, 121-126. 